\title{
Nonequilibrium statistical mechanics of the evolution of dislocation structures
}

\author{
A. H. W. Ngan \\ Dept. Mechanical Engineering, The University of Hong Kong, Pokfulam Rd., Hong Kong, People's Republic of China
}

Robb Thomson*

250 Alameda, Apt. 515, Santa Fe, New Mexico, 87501 USA

(Received 24 August 2006; revised manuscript received 13 November 2006; published 11 January 2007)

\begin{abstract}
We develop a form of nonequilibrium statistical mechanics designed to be applicable to the evolution of dislocation structure (or patterning) during metal deformation. The formalism can be applied both to time independent relaxed dislocation systems as well as to the time dependent relaxation itself. One specific application is to a simplified version of the "equilibrium" relaxed state, where we show that an effective temperature can be defined in terms of the noise in the system (back stress fluctuations). As the noise is decreased, varying degrees of order appear. In a second application to a simple two-dimensional (2D) dislocation computer model, we show how to obtain an effective time dependent free energy and temperature from the ensemble driving forces developed in earlier work. In this model, too, the underlying physics relates to a competition between the noise and energy. And, finally, we show that the behavior of the Boltzmann $\mathrm{H}$ function for the same 2D computer model can be tied to the rather complex physics of the evolving time dependent structure.
\end{abstract}

DOI: 10.1103/PhysRevB.75.014107 PACS number(s): 61.72.Bb, 61.72.Cc, 61.72.Lk, 05.20.Gg

\section{INTRODUCTION}

The study of nonequilibrium statistical mechanics goes back at least to the work of Boltzmann. In modern times, it has morphed into linear response transport theory. ${ }^{1}$ But in these studies, the underlying system is always thermal, and the local temperature is a well defined quantity, so the system is at best near thermal equilibrium. However, more recently, studies have addressed the problem of the nonequilibrium statistical mechanics of time dependent nonlinear dynamic systems. In these systems, the phase space becomes fractal, and the entropy can become singular. ${ }^{2-4}$ This field is still very much a work in progress, so there is no agreement on the form of an adequate general nonequilibrium statistical mechanics for all physical systems. In a different direction, there have also been successful attempts to define an effective temperature in certain simple highly dissipative systems. ${ }^{5,6}$ Our focus in this paper will be on the evolution of dislocation partially ordered structures during deformation. ${ }^{7}$ Berdichevski ${ }^{8}$ has very recently developed a statistical approach to a plane strain system composed of screw dislocations based on more general prior ideas about nonequilibrium systems. (See that paper and his book ${ }^{9}$ for further references.) And, finally, Edwards and Grinev ${ }^{10}$ have proposed that the approach to the steady state of a granular material can be studied as a nonequilbrium "ergodic" system, which one of us ${ }^{11}$ has applied to the problem of structure evolution in a dislocation system.

Picking up on the approach of Edwards and Grinev, ${ }^{10}$ we will show that Gibbsian statistical mechanics can be extended to an interesting class of nondynamic problems, with ideas which are quite different from the approaches being explored for the nonlinear dynamic case noted above. ${ }^{2-4}$ They also differ in various ways from the approach of Berdichevski. ${ }^{8}$ The idea of an ensemble of systems will be central to our thoughts, and for definiteness, this ensemble can be thought of as a set of computer simulations of a relaxation problem. The variables of the theory, the energy, etc., will be simulation averages. The dislocation relaxation problem and the evolution of partially ordered dislocation structures during the relaxation is the one of special interest to us, so that is where our focus will be. But we note that the theory is wider than that specific application.

We will show that the analysis leads to a particularly happy intuitive understanding of the dislocation problem wherein the (effective) temperature of the theory is associated with noise in the system which the physics of the dislocation system demands. This noise supplies the fluctuations that carry the system away from its ground state in much the same way that energy fluctuations of a thermal sort do for thermal systems. This will lead in our particular case to partially ordered structures in the dislocation system that are created by the balance between the noise generated disorder and the energetic tendency to order.

We will find that the analysis is very straightforward and utterly simple on both logical and analytic levels.

The history of attempts to understand the evolution of partially ordered dislocation cell walls as a metal deformed into stage III $^{7}$ illustrates the general point that the evolution of nonequilibrium systems has been difficult to grasp. The partial ordering in the metal systems is so suggestive of a classic phase change that attempts to apply ordinary statistical thermodyamics to the dislocation system were made very early. ${ }^{12}$ But the fact that the ordering energy per lattice site of a dislocation is of the order of $\mathrm{eV}$, whereas the thermal ordering energy is only of order $1 / 40 \mathrm{eV}$ is irrefutable evidence that the dislocation system is in fact far from thermal equilibrium, and cannot be understood on the basis of classic statistical thermodynamics.

A much more interesting approach to the dislocation evolution problem was developed by Hahner, ${ }^{13,14}$ who proposed that the transition to partially ordered walls was a noise induced transition. He developed convincing evidence that noise (the back stress) was in fact a serious factor, when he and co-authors showed ${ }^{15}$ that the observed cell structure was fractal, a result that was a direct consequence of the random- 
ness of the noise. But Hahner's noise-based analysis does not incorporate energetic forces (except for annihilation of unlike dislocations), and this violates a very deep seated intuitive sense that the ordering problem must involve energetic forces as well as noise. Also, the theory does not distinguish between edge and screw dislocations, whereas it can be easily demonstrated that screw dislocations of a single kind cannot form walls. Thus the Hahner theory must be considered at best incomplete.

Thomson et al. ${ }^{16}$ studied computer simulations of a very simple system of edge dislocations which exhibited partially ordered vertical walls. They showed that their twodimensional (2D) dislocation computer model could be understood in terms of opposing ensemble driving forces derived from the energy and the noise, and that the final state was a balance between these two. This finding seems to vindicate the idea that the ordering must involve the energy as well as the noise operating on the same logical footing.

$\mathrm{Ngan}^{11}$ showed that the results of Hahner and Thomson and co-workers correspond to two limiting cases.

In this paper, we will first modify Ngan's previous approach in order to develop a more satisfying but still analytic theory of dislocation wall evolution based on an approximate treatment of the actual edge dislocation force law. We will then show how to define an effective free energy and temperature for the 2D simulations of Thomson et al., and explore the time dependence of the Boltzmann $H$ function for that model. Finally, we will demonstrate that the noise is the seat of the effective temperature in the system for both the analytic and 2D computer models and that the effective temperature thus represents the same physical reality in both cases.

\section{A NONEQUILIBRIUM GIBBSIAN STATISTICAL MECHANICS}

We start with an ensemble of physical systems which has been constructed with a flat a priori probability distribution. But we specifically exclude Hamiltonian dynamics from our consideration. The flat a priori distribution for a set of computer simulations follows from the fact that the set of simulations is itself a flat distribution, if the systems involved are stochastically chosen.

For any such ensemble, a set of ensemble variables, such as the number of dislocations, the elastic interaction energy between dislocations, etc., is defined and measured.

The total number of all possible states of any such ensemble will be called

$$
\Omega=\sum_{s} \Omega_{s}
$$

where $\Omega_{s}$ is the number of states of the ensemble for which the ensemble variable $s$ has a specific value. For example, for an ensemble with total interaction energy, $E_{\text {ens }}$, the number of states possible in the ensemble with energy $E_{p}$ is $\Omega_{E_{p}}$. Because of the flat a priori ensemble distribution, the normalized probability for an ensemble to be in the state $s$ is

$$
P(s)=\frac{\Omega_{s}}{\Omega}
$$

From these (not necessarily equilibrium quantities), we define the Boltzmann entropy, and the Boltzmann $H$, as

$$
\begin{gathered}
\frac{S_{B}}{k}=\ln \Omega, \\
H=\langle\ln P(s)\rangle_{s}=\int P(s) \ln (P(s)) d s .
\end{gathered}
$$

The constant $k$ is analogous to the Boltzmann constant, but in this case is simply a constant which gives the appropriate dimensions to $S_{B}$. The integral in the second equation is over the range of the variable $s$.

The Boltzmann $H$ theorem states that under the very general conditions when detailed balance is satisfied (in either quantum or classical mechanics), $d H / d t \leq 0$. See Reif, Appendix A12. ${ }^{17}$

The equivalence between the Boltzmann entropy, $\mathcal{S}_{B}$, and the Boltzmann $H$ is demonstrated by introducing the familiar function, $W$, which is the count of the number of distinguishable states accessible to the ensemble. That is, with Gibbs, we make a table of all the distinguishable states of each system of the ensemble, labeling them with an index, $s$, and count the number of systems in the ensemble with distinct values of the energy $E_{s}$. This results in a distribution function, $n_{s}$, for the energy as a function of $E_{s}$. If there are a total of $M$ systems in the ensemble, then the number of distinguishable states, $W$, accessible to the ensemble is

$$
W=\frac{M !}{\prod_{s} n_{s} !},
$$

with the Stirling formula,

$$
\ln W=M \ln M-\sum_{s} n_{s} \ln n_{s} .
$$

Since $S_{B}=k \ln W$, and the normalized $P_{s}=n_{s} / M$ for a general distribution $n_{s}$,

$$
\frac{S_{B}}{k M}=\ln M-\sum_{s} P_{s} \ln \left(M P_{s}\right)=-\sum_{s} P_{s} \ln P_{s}=-H .
$$

The relationship $S_{B} / k M=-H$ fully connects the Boltzmann $H$ theorem and $\mathcal{S}_{B}$ in the second law and is valid for the fully general time dependent nonequilibrium system.

In the special case when the total ensemble energy, $E_{\text {ens }}$, is known to be a fixed time-independent constant, then the standard Gibbs argument asks for the condition of maximum probability, under the constraints that the total number of systems in the ensemble, $M$, and the total energy of the ensemble, $E_{\text {ens }}$, are both fixed. That is, the total ensemble is isolated. We follow Gibbs in not assuming that the energy is an invariant from system to system (microcanonical ensemble), because in the 2D dislocation computer model, to be explored later, the energy fluctuates from simulation to simulation. But, of course, the average energy of the ensemble is a well defined quantity. Thus the ensemble of in- 
terest is the Gibbs canonical ensemble. Then by standard arguments for the constrained maximum of $W$,

$$
\begin{gathered}
n\left(E_{s}\right)=e^{-\beta E_{s}-\alpha}, \\
\sum_{s} e^{-\beta E_{s}-\alpha}=M, \quad \sum_{s} E_{s} e^{-\beta E_{s}-\alpha}=E_{\mathrm{ens}} .
\end{gathered}
$$

By the same standard arguments, it then follows that

$$
\begin{gathered}
Z=\sum_{s} e^{-\beta E_{s}}, \quad F_{\text {ens }}=-\frac{1}{\beta} \ln Z, \\
\frac{S_{\text {ens }}}{k}=\ln Z-\beta \frac{\partial \ln Z}{\partial \beta},
\end{gathered}
$$

where $Z$ is the familiar partition sum and $F_{\text {ens }}$ is the ensemble free energy. As noted earlier, the constant, $k$, is analogous to the Boltzmann constant, but is here simply an arbitrary constant that sets the dimension of $S_{\text {ens }}$. Clearly, in this analysis, once the flat a priori ensemble distribution is established, all the standard statistical mechanics relations follow in lock step.

We note the important distinction between the time independent fully equilibrium Eqs. (7) and (8), and the time dependent equations, Eq. (3), valid for true nonequilibrium. We will find occasion to study both the time independent equilibrium case, for example, when a time dependent system relaxes to equilibrium, as well as fully time dependent nonequilibrium situations, for example while a relaxation is still taking place.

The only new thing in the equilibrium analysis is the interpretation of the effective temperature, $1 / \beta$, since the standard connection to the gas law and the absolute temperature scale is not made. But it is still a property of the analysis that the temperature introduces energy fluctuations into the system and that, at zero temperature, the system would be in the ground state (possibly degenerate) of global minimum energy, consistent with the energy constraints placed on the system. The point to be emphasized here is that the temperature for this nonthermal case has all the meaning of a generalized thermodynamic temperature, except that the "heat bath" of the nonthermal system (the energy constraint) is not the same as that for the Kelvin absolute temperature. We return below to a further discussion of these points.

We note one more general property of such a nonthermal equilibrium ensemble. In the limit of very large $M$, the probability distribution for the time independent equilibrium system exhibits the same kind of extremely narrow Gaussian maximum one is familiar with in thermal systems. This is such an important property that we repeat the steps of the familiar argument here. ${ }^{1}$

We work with the normalized probability distributions, $P\left(E_{s}\right)$ of Eq. (2), but restricted to the equilibrium case, where one can write

$$
P\left(E_{s}\right)=C \Omega\left(E_{s}\right) e^{-\beta E_{s}},
$$

where $C$ is a normalization constant. As in standard statistical mechanics, the maximum in $P$ arises because of the sharply increasing $\Omega\left(E_{s}\right)$, and the decreasing exponential.
We now expand $\ln P\left(E_{s}\right)$ about its maximum value. At the maximum, of course, $d \ln P / d E=0$, and the first term in the Taylor expansion is the quadratic. So if $2 / \eta$ $=-d^{2}(\ln P) / d E^{2}$ at the maximum, then

$$
\begin{gathered}
\ln P=\ln P_{0}-\left(E_{s}-E_{0}\right)^{2} / \eta+\cdots, \\
P\left(E_{s}\right)=P_{0} \exp \left\{-\frac{\left(E_{s}-E_{0}\right)^{2}}{\eta}\right\}+\cdots .
\end{gathered}
$$

Explicit computation of $\eta$ gives

$$
\eta=-\frac{1}{2} \frac{d \beta}{d E_{0}} .
$$

That is, the width of the peak is proportional to the effective heat capacity of the system.

The Gaussian form of the energy peak for a system can be used to test if an unknown a priori ensemble distribution is flat.

\section{STATISTICAL MECHANICS OF DISLOCATION STRUCTURES: ANALYTIC AND EQUILIBRIUM}

The central purpose of this paper is to explore noise in the dislocation system, but that noise is quite different from noise in other familiar systems and, before digging into further analysis, it is important to characterize the source and physics of the dislocation noise. When a $2 \mathrm{D}$ edge dislocation system with a single Burgers vector relaxes, it seeks the global energy minimum, which would correspond to lining all the $N$ dislocations up in a smooth single vertical wall with a constant vertical density of dislocations in the wall. But the system suffers from two sources of frustration that never allow such a global minimum to be realized. The first is that the dislocations are randomly placed on slip planes, and climb is not allowed, or at least only under strong constraint. This means that the minimum energy vertical wall has a random variation in the vertical dislocation density in the wall so the energy is higher than the global minimum. The second frustration is subtler, and is associated with the peculiar angular dependence of the glide force between two dislocations. For a given pair, if they lie oriented within a sector $\pm 45^{\circ}$ about the slip plane, the force is repulsive; otherwise, the force is attractive. For many dislocations in an attractive orientation, the configuration becomes a vertical wall. In the repulsive orientation, of course, the dislocations do not line up in vertical walls. As additional dislocations in both orientations are added, the situation becomes very complex, but the minimum energy for the system will not consist of a single vertical wall of dislocations. Instead, some local groups of dislocations will form wall configurations, while others will maximize their relative distances from one another.

These two sources of frustration lead to a fluctuating back stress on the slip plane at the relaxed structure which constitutes a resistance to ordering. In the micromechanics of the system, to achieve a completely perfect vertical wall, some dislocations would have to surmount an energy barrier. We term this fluctuating back stress the noise in the system and, 
in these terms, the actual system minimum corresponds to a balance between the global energetics favoring wall formation and the noise that resists it.

The standard approach in chemical statistical thermodynamics would be to introduce a specific assumption for the energy of the dislocation system and compute the appropriate partition sum. But in the dislocation problem, the interaction energy between pairs is not only long ranged, but angular dependent, which leads to a degree of complexity never addressed in the field of chemical thermodynamics. In the face of this difficulty, instead of attempting to compute the partition sum for a multiparticle system, $\mathrm{Ngan}^{11}$ focuses on the energy distribution among the lattice sites of the system expressed in terms of a local density of dislocations. This amounts to the adoption of a field method for the problem, where one works with the more primitive distribution functions, $P\left(E_{s}\right)$ in Eq. (9).

\section{A. Analytic energy distribution functions}

We proceed from the energy of a particular system of the ensemble in terms of its individual energy states,

$$
E_{s}=\sum_{i} \epsilon_{s i}
$$

where in the field approach mentioned above, the index pair, $\{s i\}$, refers to the $i$ th lattice site of the sth system in the ensemble. The definition of the entropy of the system follows from Eq. (3), where the sum over the index, $s$, means the sum over the energy probability distributions of the systems of the ensemble. $S_{\text {ens }}$ is the entropy of the entire ensemble,

$$
S_{\mathrm{ens}}=-\sum_{s} P\left(E_{s}\right) \ln P\left(E_{s}\right) .
$$

The distribution functions for independent systems can be written as a product of normalized distribution functions for the individual systems, $p\left(\epsilon_{s i}\right)$

$$
P\left(E_{s}\right)=\prod_{i} p\left(\epsilon_{s i}\right)
$$

The free energy of the ensemble is also decomposed into the individual free energies of the systems composing the ensemble,

$$
\begin{aligned}
F_{\mathrm{ens}} & =E_{\mathrm{ens}}-\theta S_{\mathrm{ens}}=\sum_{s} F_{s}, \quad \theta=\frac{k}{\beta}, \\
F_{s}=E_{s}-\theta S_{s} & =\sum_{i}\left[\epsilon_{s i} p_{\epsilon}\left(\epsilon_{s i}\right)+\theta p_{\epsilon}\left(\epsilon_{s i}\right) \ln \left(p_{\epsilon}\left(\epsilon_{s i}\right)\right)\right] \\
& =\int\left[\epsilon_{s i} p_{\epsilon}\left(\epsilon_{s i}\right)+\theta p_{\epsilon}\left(\epsilon_{s i}\right) \ln \left(p_{\epsilon}\left(\epsilon_{s i}\right)\right)\right] d \epsilon_{s i},
\end{aligned}
$$

where the individual system entropies are $S_{\text {ens }}=\Sigma_{s} S_{s}$. Writing $S_{\text {ens }}$ as the sum of the individual system entropies can only be done at equilibrium and, for dynamical systems, involves a subtle argument about the difference between coarse grained and fine grained distributions. ${ }^{18}$ We expect no such difficul- ties in our nondynamic relaxing dislocation system because there is no Poincaré cycle in these systems, and the distribution function is always "coarse grained" with a well defined time arrow.

In the last equation, the discrete variables have been converted to continuous functions and $p_{\epsilon}\left(\epsilon_{s i}\right)$ is the normalized probability that a lattice site $i$ has the energy, $\epsilon_{s i}$. Since we wish to work with dislocation lattice densities, $\rho$, a new probability density function, $p_{\rho}(\rho)$ must be defined by

$$
p_{\rho}(\rho) d \rho=p_{\epsilon}(\epsilon) d \epsilon
$$

Here, $\rho$ is the normalized density defined as $\rho=\rho^{\prime} / \bar{\rho}$, where $\rho^{\prime}$ and $\bar{\rho}$ are the absolute and mean densities, respectively.

The physics of the dislocation system is fully determined when the energy of a lattice site is specified in terms of the local density of dislocations. In addition, we specify that the density distribution is normalized. The problem is then to determine the probability distribution, $p_{\rho}$. Since the probability distribution function is so far arbitrary (within the constraint of a fixed number of dislocations), the probability density of interest is the one at mechanical equilibrium, which means the one that minimizes the free energy function with respect to the probability distribution function. (As in standard statistical thermodynamics, for a canonical ensemble at equilibrium, the free energy is a minimum.)

The only conceptual question remaining is that the subscript, $s$, remains on system functions, and since the system is canonical, these quantities vary from system to system in the ensemble. Final results are obtainable for the ensemble, when the system quantities are summed over, e.g., $F=\Sigma F_{s}$. In practice, it is often possible to ignore the difference between $F_{s}$ for a particular system and $F / M$, because for a large ensemble, $M$, the relative fluctuations become very small. With this assumption, in the following, we drop the subscripts $s$.

Thus we engage, once again, in a constrained minimization process, but this time for the free energy of the system,

$$
\frac{\delta F}{\delta p_{\rho}}=0,
$$

with the constraint that the number of dislocations is fixed,

$$
\int \rho p_{\rho}(\rho) d \rho=1
$$

$\left(\delta F_{s} / \delta p\right)$ is the variational derivative with respect to the function $p_{\rho}$. If it is possible to write a relation between the energy at a lattice site and the local dislocation density at that site, then the mathematical procedure laid out is well defined.

In his previous work, $\mathrm{Ngan}^{11}$ explored a problem that is equivalent to the process above except he assumed a microcanonical ensemble. That is, he considered a single system with fixed energy. We will wish to work with an ensemble with fluctuating energy, so the canonical ensemble is the appropriate one for the dislocation problem. This introduces a slight change in the analysis, but no practical difference in the final result. 
He introduced two different energy functions: One appropriate for low densities of dislocations and a second appropriate to high density, where the dislocations are already in walls. The one for low density was

$$
\epsilon^{(1)}\left(\rho_{i}^{\prime}\right)=\frac{a \mu b^{2}}{4} \ln \left(\frac{\rho_{i}^{\prime}}{\rho_{\min }}\right),
$$

where the prelogarithmic factor is a constant and $\rho_{\min }$ refers to the outer cutoff radius for the dislocation distribution. For high density, he considered the function

$$
\epsilon^{(2)}\left(\rho_{i}^{\prime}\right)=\frac{a \mu b^{2}}{8 \pi} \ln \left(\frac{\rho_{\max }}{\rho_{i}^{\prime}}\right),
$$

where $\rho_{\max }=1 / b^{2}$. Note that these two energy functions are on a per unit dislocation length basis in a local environment with density $\rho_{i}^{\prime}$.

In this paper, we use these energy functions in a very different way than in his earlier paper. Our strategem, here, is to introduce a distribution composed of two independent subdistributions of dislocations; one which corresponds to pairs oriented in the repulsive manner and a second which corresponds to pairs oriented in the attractive manner. Because of the physical symmetry, we will assume that half of the dislocations, $N_{d} / 2$, are in the repulsive distribution and half in the second. The energy function for the first repulsive distribution is simply Ngan's energy expression for the low density limit, Eq. (19), where $\epsilon^{(1)}$ is the energy for the repulsive density and $\rho_{1}^{\prime}$ refers to the repulsive density function. The energy function for the attractive distribution is Ngan's expression for the high density limit, Eq. (20), where $\epsilon^{(2)}$ refers to the energy of the attractive distribution density function, $\rho_{2}$. Even though, in his earlier paper, these two functions were meant to describe a single distribution in the two physical limits of low and high density, they are also the appropriate functions for repulsive and attractive subdistributions, respectively.

This strategem is, of course, highly arbitrary and contains serious energetic inconsistencies relative to real dislocations. For example, the model does not include interactions between dislocations in different distributions, even though, in reality, these interactions are very important. Indeed, it is these interactions that form the basis for the frustration in the system, and which leads in turn to noise. A second inconsistency is that no exchange from one distribution to the other is allowed when pairs switch their character during motion. Nevertheless, because the two-distribution model distinguishes repulsive interactions from attractive ones, and is analytic, it is a very attractive toy model to explore. The physical interpretation is that the two different subsystems coexist on the same lattice at the same time.

\section{B. Effective temperature}

As noted previously, through the temperature, $1 / \beta$, the system is subject to fluctuations. At the equivalent of absolute zero temperature, each subsystem will sit in its global energy minimum. In the repulsive case, that will be a completely ordered state with each dislocation equidistant from every other on a regular dislocation lattice. In the attractive case, that will be a state with the system in a single ordered wall stretching from the upper boundary to the lower. (In both cases, there is no injunction against climb, so there is no constraint to the system achieving an absolute global minimum.)

But, physically, the system cannot achieve the state of lowest global minimum, because of the two forms of frustration in the model: The constraint of no climb and the angular dependent force law. Both forms of frustration lead to noise in the sense of fluctuations in the back stress in the system. Of course, there is a minimum back stress noise level in even a completely ordered wall, and all other noise is measured against this minimum value. Since the noise contributed by the climb, constraint is small compared to that contributed by the angular dependent forces; we will simply designate the "noise" as the noise generated by the angular dependent force law.

In the two-distribution model, this noise is generated by the forces between a dislocation in the repulsive distribution and a dislocation in the attractive distribution. Setting the temperature to a positive value introduces the noise in the system that prevents complete global ordering between the two populations, and thereby injects the frustration physics in an ad hoc manner. With this perspective, it is appropriate that $\beta$ is the same in the two distributions, since interactions between a repulsive dislocation with an attractive one is exactly the same as the interaction of an attractive dislocation with a repulsive one. In the earlier language, this means the two distributions are, in fact, in contact with the same heat bath.

Since the distributions are assumed to be completely independent of each other, the total elastic energy of the two dislocation populations for a particular system in the ensemble is

$$
\frac{E_{s}}{N_{d}}=\int_{0}^{\infty} \epsilon_{1} \rho_{1} p_{1}\left(\epsilon_{1}\right) d \epsilon_{1}+\int_{0}^{\infty} \epsilon_{2} \rho_{2} p_{2}\left(\epsilon_{2}\right) d \epsilon_{2}+\epsilon_{0}
$$

Here $\epsilon_{1}$ and $\epsilon_{2}$ are given by Eqs. (19) and (20), respectively, so that the two integrals represent the interaction energies between dislocations, and $\epsilon_{0}$ is an unimportant constant term representing the self-energies of the individual dislocations. In Eq. (21), $\rho=\rho^{\prime} / \bar{\rho}$, where $\bar{\rho}$ is the mean density in each population, and the appropriate subscripts to the variables are added when applying to a particular subdistribution. The entropy $S_{s}$ is given by Eq. (15)

$\frac{S_{s}}{N_{d}}=-k_{1} \int_{0}^{\infty} p_{1}\left(\epsilon_{1}\right) \ln \left[p_{1}\left(\epsilon_{1}\right)\right] d \epsilon_{1}-k_{2} \int_{0}^{\infty} p_{2}\left(\epsilon_{2}\right) \ln \left[p_{2}\left(\epsilon_{2}\right)\right] d \epsilon_{2}$.

In the light of Eqs. (19) and (20), normalization with the energy terms can be achieved by setting the two Boltzmannlike constants to be

$$
k_{1}=\frac{a \mu b^{2}}{4}, \quad k_{2}=\frac{a \mu b^{2}}{8 \pi}=\frac{k_{1}}{2 \pi} .
$$




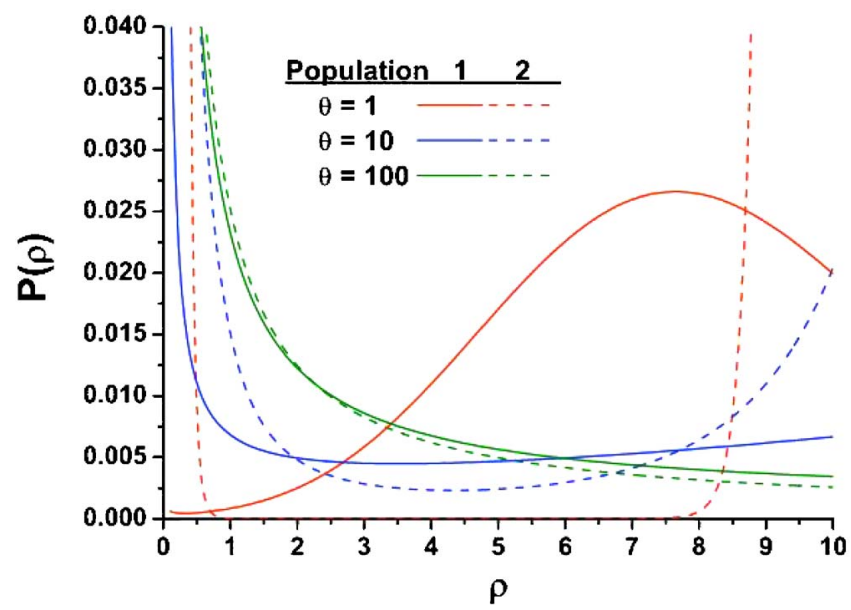

FIG. 1. (Color online) Probability density functions for the two noninteracting populations for different values of the effective temperature, $\theta$.

The effective temperature, $\theta$, is defined from the effective free energy function in Eq. (15). Note that the two populations are linked by a common temperature $\theta$, interpreted as the retained noise level in the system.

Following the analysis carried out in Ngan's earlier paper, minimization of $F_{s}$ in Eq. (15) with $E_{s}$ and $S_{s}$ defined respectively in Eqs. (21) and (22) leads to

$$
\begin{gathered}
p_{1}\left(\rho_{1}\right)=\frac{A_{1}}{\rho_{1}} \exp \left[\frac{\rho_{1}}{\theta}\left(c_{1}-\ln \rho_{1}\right)\right], \\
p_{2}\left(\rho_{2}\right)=\frac{A_{2}}{\rho_{2}} \exp \left[\frac{2 \pi \rho_{2}}{\theta}\left(\ln \rho_{2}-c_{2}\right)\right] .
\end{gathered}
$$

The constants, $c$, in Eq. (24) are set to enable satisfaction of the auxiliary constraint

$$
\int_{0}^{\infty} \rho p(\rho) d \rho=1
$$

for each population. The constants, $A$, are normalization constants. As discussed in Ngan's earlier paper, because the force law is attractive, the probability function for population 2 diverges with $\rho_{2}$ and so, in practice, the upper limits in the constants in Eq. (25) have to be replaced by a maximum density (e.g., 10).

Figure 1 shows the probability functions for the two populations evaluated at three different temperature values. It can be seen that at a small temperature value $(\theta=1)$, the two populations adopt behaviors governed mainly by their force laws in Eqs. (19) and (20), namely, population 1 tends to adopt a uniform distribution [with a unimodal $\left.p_{1}\left(\rho_{1}\right)\right]$ in which dislocations are kept furthest apart from one another, and population 2 adopts a segregated distribution in which high and low density domains coexist. At increasing temperatures (e.g., $\theta=100$ ), however, the two populations tend to behave the same way, with a fractal-type power law density distribution. The force law therefore does not matter much in determining pattern formation at high noise levels.

\section{2D COMPUTER SIMULATIONS}

In an earlier computer simulation study of edge dislocations with a single Burgers vector in $2 \mathrm{D}$, Thomson et al. ${ }^{16}$ found an ensemble driving force for ordering due to energetics, and another disordering force due to the noise. They showed that the relaxed equilibrium state corresponded to a balance between these two ensemble forces. The purpose of this section is to extend the earlier treatment by integrating the ensemble force balance at the relaxed equilibrium state to find a phenomenological effective free energy for the $2 \mathrm{D}$ simulation system and, from that, the effective temperature of this 2D discrete system. In addition, we will also extend the earlier work by exploring the time dependence of the Boltzmann $\mathrm{H}$ function (i.e., the entropy) of the system as it relaxes under the action of the force laws. Finally, we will show how the 2D discrete simulations relate to the analytic model of the previous section.

\section{A. Phenomenological integration of the ensemble driving forces}

In the simulations of Thomson et al. ${ }^{16}$ the sample is a $2 \mathrm{D}$ square cell 1000 sites on a side containing $N$ straight parallel dislocations vertical to the sample cross section. The Burgers vectors are all in one direction, $X$, and all of a single sign. (In a true metal, this would correspond to a bent crystal, but we do not allow the lattice to rotate. This feature makes the model highly nonphysical, but we are free to study any "toy" model we like, so long as it is self consistent.) The dislocations interact with the fully angular dependent 2D elastic interaction.

In the modeling, an initial distribution is generated randomly and the system is allowed to relax under the interaction forces. Specifically, in a simulation, the force on each dislocation in turn is computed. If the absolute value of the force on the dislocation is greater than a specified value (the analogue of the Peierls stress), that dislocation is then moved one lattice spacing in the direction of the force. The force computation is repeated ten times for each new position, and then the computer moves on to the next dislocation in the list in repeated relaxation cycles till the system is perceived to have converged. Note there is no kinetic energy in the system, because that is quenched out every time a dislocation is moved. After the system has relaxed for a specified number of cycles, a new simulation is performed with a new starting random configuration for a specified number of simulations. The totality of simulations constitutes an ensemble. Ensemble averages for the interaction energy, etc. can be computed for each relaxation cycle, so the relaxation history of the system can be examined.

It is found that for small Peierls stress, the relaxation is complex, with one fast relaxation, followed by a second much longer relaxation. We will identify the "time" with the total count of relaxation cycles from the beginning of a simulation.

The ensemble functions computed from the simulations are the total interaction dislocation energy, $\mathcal{E}$, the noise, $\mathcal{R}$, which is the average of the square of the stress at each point on a slip plane, and the wall correlation function, $\mathcal{C}$. The wall 
correlation function is simply the vertical pair correlation function. (This is a small deviation from the wall correlation function defined in Thomson et al. $\left.{ }^{16}\right)$. The ensemble functions are all functions of the cumulative number of relaxation cycles in a simulation, $t$, and on the total number of dislocations in the system, $N_{d}$. If the Peierls stress is above a critical value, all the ensemble functions of the early system response are found to be simple exponentials,

$$
\begin{aligned}
& \mathcal{E}\left(N_{d}, t\right)=e_{0}\left(N_{d}\right) \exp \left(-t / \alpha\left(N_{d}\right)\right)+e_{1}\left(N_{d}\right), \\
& \mathcal{R}\left(N_{d}, t\right)=r_{0}\left(N_{d}\right) \exp \left(-t / \zeta\left(N_{d}\right)\right)+r_{1}\left(N_{d}\right), \\
& \mathcal{C}\left(N_{d}, t\right)=c_{0}\left(N_{d}\right) \exp \left(-t / \gamma\left(N_{d}\right)\right)+c_{1}\left(N_{d}\right) .
\end{aligned}
$$

Note that here $c_{0}<0$, while all other constants $>0$, i.e., $\mathcal{E}$ and $\mathcal{R}$ decrease with $t$ while $\mathcal{C}$ increases with $t$ as relaxation proceeds. It is also found that the initial relaxation times for $\mathcal{E}$ and $\mathcal{R}$ were roughly equal. $\mathcal{C}$ has a much longer relaxation time than $\mathcal{E}$ and $\mathcal{R}$, so that wall formation continues long after the fast relaxation for $\mathcal{E}$ and $\mathcal{R}$ have ended. The long relaxation tails observed for $\mathcal{E}$ and $\mathcal{R}$ after the fast relaxation has ended at $t \approx 2 \alpha$, and when the Peierls stress is small( $<6 \times 10^{-4} \mu$ ), are associated with the longer relaxation time for wall formation. These long tails are slow power laws, $\mathcal{E}$ $\sim t^{-2}$ and $\mathcal{R} \sim t^{-2}$, instead of exponentials. The fast relaxation stages appear to be associated with relatively gross rearrangements of the dislocations, and the longer tails with small energy changes associated with relatively subtle later stages of wall formation. In this subsection (but specifically not in subsection D), to keep the model as simple as possible, we will choose the value of the Peierls stress to restrict the system within the fast relaxation regime of the energy, so that the ensemble functions are describable in terms of a single exponential function. In addition to this rather heuristic use of the idea of a Peierls stress, we will also use it below as a control parameter to explore the physics of the relaxation process.

An ensemble driving force, $\mathcal{F}_{\mathcal{E}}\left(N_{d}, t\right)$, for ordering is defined as

$$
\begin{gathered}
\mathcal{F}_{\mathcal{E}}\left(N_{d}, t\right)=-\left(\frac{\partial \mathcal{E}\left(N_{d}, t\right)}{\partial \mathcal{C}\left(N_{d}, t\right)}\right)_{N}=-\frac{e_{0} \gamma}{c_{0} \alpha} e^{-t / \phi}, \\
\phi=\frac{\alpha \gamma}{\alpha+\gamma},
\end{gathered}
$$

where the second line is valid for the fast relaxation regime at fixed $N$. We find that the relaxed state is determined by

$$
\mathcal{F}_{\mathcal{E}}\left(N_{d}, t=2 \alpha\right)=\mathcal{F}_{\mathcal{R}}\left(N_{d}, t=2 \alpha\right)=\kappa \mathcal{R}\left(N_{d}, t=2 \alpha\right) .
$$

The time, $2 \alpha$, is a convenient time at the end of the fast relaxation regime, which we define to be the relaxed state.

Using this convention for the relaxed state, it is found that $\kappa$ is a fixed constant for different $N_{d}$. That is, $\kappa$ depends only on the force law, and not on $N_{d}$. But we note a subtle point. For two relaxed states of different Peierls stress, $\kappa$ is different. As the Peierls stress increases, $\kappa$ decreases.

\section{B. Phenomenological time dependent free energy function}

It is possible to integrate the force balance equation to obtain a function whose minimum corresponds to the force balance at the relaxed state. Such a function, the effective free energy function, is

$$
\mathcal{G}_{p}=\mathcal{E}-\theta \mathcal{S}_{p},
$$

such that at the relaxed state, $\mathcal{G}_{p}$ is a minimum,

$$
\left[\frac{\partial \mathcal{G}_{p}}{\partial \mathcal{C}}\right]_{N_{d} t=2 \alpha}=-\left[\mathcal{F}_{\mathcal{E}}+\theta \frac{\partial \mathcal{S}_{p}}{\partial \mathcal{C}}\right]_{N_{d}, t=2 \alpha}=0 .
$$

Comparing the equilibrium conditions in Eq. (28) and Eq. (30),

$$
\theta\left[\frac{\partial \mathcal{S}_{p}}{\partial \mathcal{C}}\right]_{N_{d}, t=2 \alpha}=\theta\left[\frac{\partial \mathcal{S}_{p}}{\partial \mathcal{R}} \frac{1}{(\partial \mathcal{C} / \partial \mathcal{R})}\right]_{N_{d}, t=2 \alpha}=-\kappa[\mathcal{R}]_{N_{d}, t=2 \alpha}
$$

This is a condition for one time only, so it can be satisfied in a number of ways, which we tailor to satisfy the physical requirements. We thus postulate that

$$
\begin{gathered}
{\left[\frac{\partial S_{p}\left(N_{d}, t\right)}{\partial \mathcal{R}\left(N_{d}, t\right)}\right]_{N_{d}}=\kappa, \quad \mathcal{S}_{p}\left(N_{d}, t\right)=\kappa \mathcal{R}\left(N_{d}, t\right),} \\
\theta\left(N_{d}\right)=-\left[\mathcal{R} \frac{\partial \mathcal{C}}{\partial \mathcal{R}}\right]_{N_{d}, t=2 \alpha} .
\end{gathered}
$$

According to the second of these equations, the effective temperature, $\theta$, is proportional to the noise, $\mathcal{R}$, at the equilibrium point, which is physically satisfying. It is defined for a time dependent process in terms of the final relaxed state. Note that the temperature is positive, because $(\mathcal{C C} / \partial \mathcal{R})$ is negative. The expression for the free energy is now fully determined, is consistent with the balance of forces at $t$ $=2 \alpha$, and is given by

$$
\mathcal{G}_{p}=\mathcal{E}-\kappa \theta\left(N_{d}\right) \mathcal{R}\left(N_{d}, t\right)=\mathcal{E}+\kappa \mathcal{R}\left(N_{d}, t\right)\left[\mathcal{R} \frac{\partial \mathcal{C}}{\partial \mathcal{R}}\right]_{N_{d}, t=2 \alpha} .
$$

For a different simulation with a different starting number of dislocations, the functional dependence on $N_{d}$ can be determined. Such a (time dependent) free energy function has all the necessary properties, and completely describes the simulation ensemble and its relaxations.

\section{Path dependence and flatness of the relaxed states}

We turn now to the application of the time dependent Gibbs analysis to the computer model. But before that can be done, two consistency checks must be run: The intermediate states must be independent of the path so the ensemble variables are unique and the time dependent system must correspond to flat ensembles.

During a relaxation process, there is only one relaxation path, so there is no question of reaching the state by different 


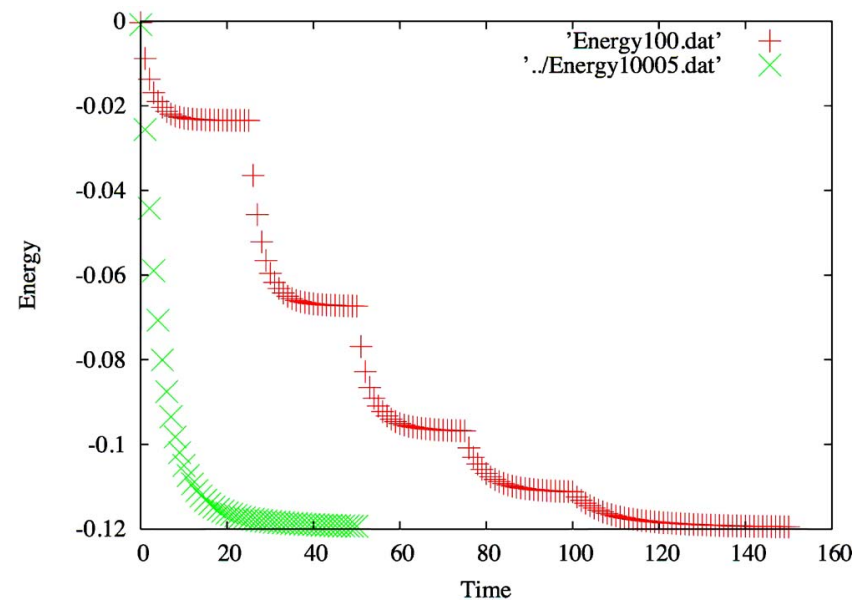

FIG. 2. (Color online) Demonstration that the final relaxed ensemble is the same independent of how it is achieved. "Energy 100.dat" is for five relaxations with varying $P$ stress, and "Energy10005.dat" is for a single relaxation with very small $P$ stress.

paths. But the final relaxed state can also be approached by a series of intermediate relaxation paths during which the Peierls stress of the system is changed during the relaxation. We show in Fig. 2 that the final state is in fact independent of intermediate Peierls stress changes, so the ensemble states and the effective free energy function, $\mathcal{G}$, etc. derived for them, are all well defined. These simulations were done in the standard way ${ }^{16}$ for 50 dislocations. In each simulation, a sequence of five values of the Peierls stress is chosen in turn, starting with a high value and ending with a very small value. Thus each sequential relaxation after the first starts with the partially ordered state of the previous relaxation.

To confirm that the $2 \mathrm{D}$ computer model represents a flat ensemble, we show that a fully relaxed system of 50 dislocations does indeed conform very accurately (for very large numbers of simulations) to a Gaussian distribution. See Fig. 3. Of course, this demonstration is only the necessary condition, and not sufficient. But, physically, the probability that a Gaussian distribution can be achieved and not correspond to a flat ensemble should be very small.

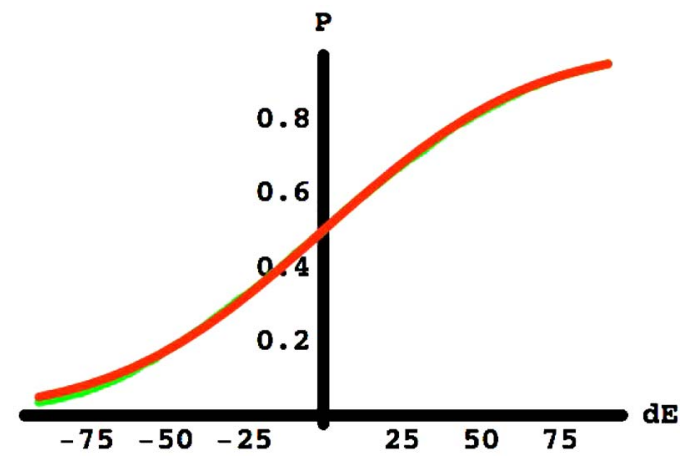

FIG. 3. (Color online) Plot of cumulative energy distribution, $P(d E)$, in the completely relaxed state for 50 dislocations. $d E$ is proportional to the energy decrement relative to the maximum of $E_{s}, d E \propto E_{s}-E_{s \max }$. Averaged over 5000 simulations. An error function fitted to the same width is plotted on the same graph. The two separate curves can hardly be resolved on the figure.

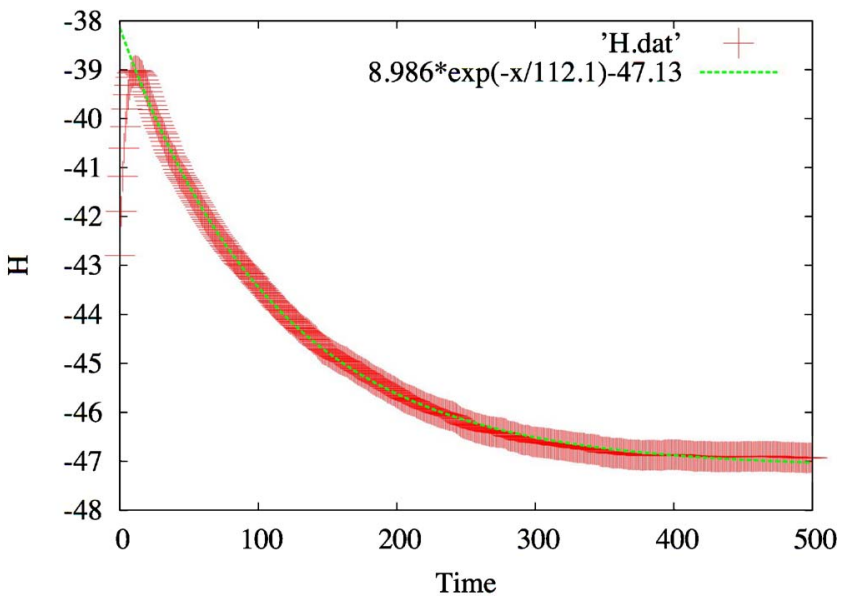

FIG. 4. (Color online) Plot of the numerical computation of $H\left(N_{d}=50, t\right)$ for 50 dislocations as a function of time for long times, showing a peak at about time $\approx 20$. Averaged over 5000 simulations. The time is in units of cumulative relaxations within a simulation. The dashed line is an exponential function with relaxation time in the long time limit of 112.1.

\section{D. $\boldsymbol{H}$ function for the computer model}

With these two consistency checks out of the way, we turn now to the direct numerical computation of the time dependent $H$ function for the 2D computer model from Eq. (3). This result is shown in Fig. 4 for 50 dislocations.

The function, $H$, is found in Fig. 4 to be an increasing exponential initially, whose initial relaxation time is $\approx 4.08$. This result apparently contradicts the $H$ theorem and implies a decreasing entropy during early relaxation. Figure 4 is plotted for a very small Peierls stress, so the relaxation can go to completion at long times. For these long times, the $\mathrm{H}$ function develops a peak and decreases slowly.

What can be happening here?

During the early fast relaxation, the rapid energy release drives the system quickly to the relaxed wall configuration. During this rapid relaxation, the effective $\Gamma$ space available to the ensemble decreases, the probability of the system decreases, and the Boltzmann entropy also decreases. In these fast relaxations, the large interaction energy changes are totally quenched after each relaxation, and this loss in potential energy is not converted to kinetic energy as it would be in a system where the energy is conserved, or in the actual physical system, where this energy is converted dissipatively into heat. In the parlance developed for such simulations, a "thermostat"19 is introduced to skim off the kinetic energy of the dislocations and/or the heat generated after each relaxation step in the computer. Of course, for the total physical system including the action of the thermostat, the total entropy of the physical system must necessarily increase during a relaxation. But the $H$ theorem is not obeyed for the quasistatic system during the fast relaxation, because the system is not isolated.

The initial rising part of the $H$ curve fully captures the changes in the system $\Gamma$ space, however. In particular, the entropy is predicted to be proportional to $\mathcal{R}$ according to Eq. (32). The early rise in $H$ has a relaxation time of 4.08 , while 
the relaxation time for $\mathcal{R}$ is $\zeta \approx 4$.9. The rough consistency between these relaxation times confirms the fact that $H$ indeed roughly tracks the increasing order in the system during the fast relaxation and its description via the phenomenological free energy.

Once the fast relaxation is over, however, the ordered state is essentially formed and the energy relaxation changes its character. At this point, instead of relaxing exponentially, the energy relaxation switches to a much slower quadratic power law decrease. (See Sec. IV A.) The correlation function, $\mathcal{C}$, does not show this two stage behavior, but instead decreases exponentially with a very long relaxation time of 110 throughout the entire process of both fast and slow relaxation. It is striking that the relaxation time for $H$ past the peak is 112 , or within numerical accuracy, the same as the relaxation time for $\mathcal{C}$.

We believe the continuing relaxation after the peak in $H$ is to be interpreted in terms of the standard decrease of $H$ for a (nearly) closed system of fixed energy. This is because the energy changes very little during the slow relaxation, compared to the exponentially decreasing $H$. When the system enters the slow relaxation regime, the major features of the ordered state have been achieved, and after this, the ensemble system explores a variety of neighboring states consistent with a nearly fixed energy. Such neighboring states might correspond to small further refinement of the walls, or to excursions of dislocations caught in shallow energy minima outside the walls. The expansion of the $\Gamma$ space associated with these low energy excursions corresponds to decreasing $H$ and increasing entropy. The fact that the relaxation times for $\mathcal{C}$ and $H$ are the same is evidence that the changes in $H$ during the slow relaxation are to be associated with subtle configuration changes. All this would be consistent with an effectively closed system, for which the Boltzmann $H$ theorem is satisfied.

This discussion can be encapsulated in an equation for the ensemble entropy, $\mathcal{S}_{\text {ens }}$,

$$
\mathcal{S}_{\text {ens }}(t)=\mathcal{S}_{\text {mean }}(t<2 \alpha)+\mathcal{S}_{\text {var }}(t>2 \alpha),
$$

where $\mathcal{S}_{\text {mean }}$ describes the fast relaxation to a kind of mean field state and $\mathcal{S}_{\text {var }}$ is the entropy of a system exploring a wider region of $\Gamma$ space. $\mathcal{S}_{\text {mean }}$ is a decreasing function of time, while $\mathcal{S}_{\text {var }}$ increases with time.

\section{E. Comparison of the noise in the analytic and computer models}

In Eq. (32) in the phenomenological approach, it is shown that the quasitemperature is related to the noise level $\mathcal{R}$ at equilibrium. In this section, we will show that a similar condition can also be obtained from the analytical model.

We begin by noting that the shear stress at a given lattice point is due largely to a nonzero gradient in the local dislocation density function. More precisely, the shear stress $\tau_{i+1 / 2}$ at the midpoint between lattice sites $i$ and $i+1$ on a slip plane is approximately given by

$$
\begin{gathered}
\tau_{i+1 / 2} \approx \frac{\mu b^{3}}{(b / 2)}\left(\rho_{i+1}^{\prime}-\rho_{i}^{\prime}\right), \\
t_{i+1 / 2}=\frac{\tau_{i+1 / 2}}{2 \mu b^{2} \bar{\rho}} \approx \rho_{i+1}-\rho_{i},
\end{gathered}
$$

where $\rho_{i}^{\prime}$ and $\rho_{i+1}^{\prime}$ are the dislocation densities at sites $i$ and $i+1$ which are assumed to be spaced $b$ apart, $\rho=\rho^{\prime} / \bar{\rho}, \bar{\rho}$ is the mean density, and $t_{i+1 / 2}$ is a normalized form of $\tau_{i+1 / 2}$. In Eq. (35), the effects of dislocations at other, more remote sites are ignored. Since the dislocation densities at $i$ and $i+1$ are distributed according to $p_{\rho}(\rho)$, the probability distribution of $t_{i+1 / 2}$ is

$p_{t}\left(t_{i+1 / 2}\right)=\int_{0}^{\infty} \int_{0}^{\infty} p_{\rho}\left(\rho_{i}\right) p_{\rho}\left(\rho_{i+1}\right) \delta\left[t_{i+1 / 2}-\left(\rho_{i+1}-\rho_{i}\right)\right] d \rho_{i} d \rho_{i+1}$,

where $\delta[\cdots]$ is the Dirac delta function. The Laplace transform of $p_{t}(t)$ in Eq. (36) is

$$
\begin{aligned}
\widetilde{p}_{t}(s) & =\int_{0}^{\infty} \int_{0}^{\infty} p_{\rho}\left(\rho_{i}\right) p_{\rho}\left(\rho_{i+1}\right) e^{-\left(\rho_{i+1}-\rho_{i}\right) s} d \rho_{i} d \rho_{i+1} \\
& =\widetilde{p}_{\rho}(s) \widetilde{p}_{\rho}(-s),
\end{aligned}
$$

where $\widetilde{p}_{\rho}(s)$ is the Laplace transform of $p_{\rho}(\rho)$ and $s$ is the transform variable. From a physical point of view, the mean of the shear stress should be zero, and indeed the same conclusion can be seen from Eq. (37), since

$$
\bar{t}=\int_{-\infty}^{\infty} t p_{t}(t) d t=-\widetilde{p}_{t}^{\prime}(0)=0 .
$$

The noise $\mathcal{R}$ in the phenomenological approach is the mean of the square of the shear stress, and so is given by

$$
\mathcal{R} \propto\left\langle t^{2}\right\rangle=\int_{-\infty}^{\infty} t^{2} p_{t}(t) d t=\widetilde{p}_{t}^{\prime \prime}(0),
$$

and from Eq. (37), $\left\langle t^{2}\right\rangle$ is given by

$$
\left\langle t^{2}\right\rangle=2\left\{\widetilde{p}_{\rho}^{\prime \prime}(0) \widetilde{p}_{\rho}(0)-\left[\tilde{p}_{\rho}^{\prime}(0)\right]^{2}\right\}=2\left[\widetilde{p}_{\rho}^{\prime \prime}(0)-1\right],
$$

since, by the normalization conditions of $\int_{0}^{\infty} p_{\rho}(\rho) d \rho=1$ and Eq. (25), $\tilde{p}_{\rho}(0)=1$ and $\widetilde{p}_{\rho}^{\prime}(0)=-1$. Assuming the equilibrium low-density distribution,

$$
\begin{gathered}
p_{\rho}(\rho)=\frac{A}{\rho} \exp \left[\frac{\rho}{\theta}(c-\ln \rho)\right], \\
\widetilde{p}_{\rho}^{\prime \prime}(0)=\int_{0}^{\infty} \rho^{2} p_{\rho}(\rho) d \rho=A \int_{0}^{\infty} \rho \exp \left[\frac{\rho}{\theta}(c-\ln \rho)\right] d \rho,
\end{gathered}
$$

and so the noise $\mathcal{R}$ at equilibrium can be evaluated as 


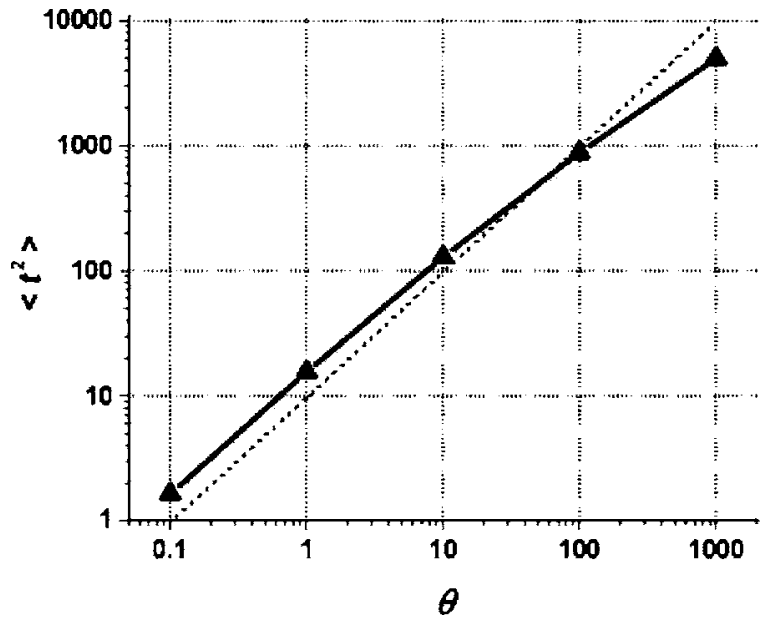

FIG. 5. Equilibrium noise at various temperatures, computed from Eq. (42).

$$
\mathcal{R} \propto\left\langle t^{2}\right\rangle=2\left\{A \int_{0}^{\infty} \rho \exp \left[\frac{\rho}{\theta}(c-\ln \rho)\right] d \rho-1\right\} .
$$

Here $A$ and $c$ are normalization constants for the $p_{\rho}(\rho)$ function at each $\theta$ value; they are therefore known functions of $\theta$, i.e., $A(\theta)$ and $c(\theta)$. Figure 5 shows the values of $\left\langle t^{2}\right\rangle$ computed at different values of $\theta$ over several orders of magnitude. It can be seen that the computed noise level at equilibrium is approximately proportional to the temperature. In the phenomenological approach, there is a factor $\partial \mathcal{C} / \partial \mathcal{R}$ in the relationship between temperature and noise as in Eq. (32). The wall correlation function $\mathcal{C}$ cannot be defined easily in the analytic approach but, in any case, exact agreement between the two approaches is not to be expected because the force laws used in the computer and analytical approaches are very different.

\section{CONCLUSIONS}

The original motivation in doing this work was to better understand the nonthermal problem of dislocation order evolution during metal deformation. After struggling with the extant ideas about the problem, we came to the firm conclusion that the question had to be addressed in terms of a competition between energy and noise in the system. We believe this is even true from a kinetic standpoint: The dissipative processes are very noisy, and they compete with energetic forces which tend toward order. This viewpoint eventually thrust upon us the fact that what was happening in this system was very similar to what happens in thermodynamics when thermal fluctuations frustrate ordering forces, for example during melting of an ordered solid. But one cannot use standard thermodynamics for this problem because of the discrepancy between thermal energies and the elastic driving forces in the deforming system. Our first step was to develop the analogue of ensemble forces in the deforming solid, and show that the ordered state was, indeed, a balance between noise and energy. But, with the current paper, we have gone to the core of statistical mechanics as developed by Gibbs and shown that his basic ideas associated with the properties of flat ensembles of systems are fully applicable to our class of nonthermal mechanical systems. Instead of using the idea of a thermal heat bath, however, for the definition of the temperature of the system, our heat bath is supplied by the noise in the system, which can be significant even at zero Kelvin temperature. This is even true when the noise is created by processes that are embedded within the physics of the system itself. In the dislocation problem, indeed, one principal cause of the noise is the angular dependent force law between dislocation pairs and the frustration this generates when many dislocations are present. Of course, in the full complexity of the deformation problem, there are other potent sources of noise, as well, such as pair formation, lock formation, point defects, etc. Studies of these additional sources of noise are left for future work.

In the current paper, we therefore begin with an extension of Gibbs statistical mechanics to the nonthermal regime suitable for the dislocation problem. In the relaxed equilibrium condition, we demonstrate the meaning of the temperature, and rules for extending the standard thermodynamic functions to apply to the nonthermal regime. It is shown that the partition sum is well defined, and that analysis analogous to standard chemical statistical thermodynamics is possible. Although, to our knowledge, our use of the Gibbs theory is new, it sidesteps the problem of the nonequilibrium statistical mechanics of nonlinear dynamic systems, which has received intense attention in recent years. (See, for example, Rondoni and Cohen. ${ }^{4}$ ) In our work, though time dependent, the system is not dynamic in the Hamiltonian sense and the $H$ function is well defined and finite.

Our first application is to an analytic but approximate treatment of equilibrium dislocation structure formation. Our approach is very approximate, but highlights in a very intuitive way the role of the dislocation temperature. This methodology results in a prediction for ordered walls as a function of the strength of the noise, and exhibits a regime for high dislocation temperatures where the structure degenerates into a fractal inhomogeneity that is akin to the "tangle" formations observed in the earlier stages of deformation.

The second application is to direct computer relaxation simulations of a 2D dislocation system composed of edge dislocations of a single sign Burgers vector. In this system, studied earlier by Thomson et al., ${ }^{16}$ the system is observed to order partially, and the ordered relaxed state is associated with a balance between ensemble energetic and noise forces. In the current paper, we show that a function analogous to a time dependent free energy function can be defined, phenomenologically, for this system, whose minimum corresponds to the relaxed partially ordered state.

We then show that during an initial fast relaxation, the Boltzmann $H$ theorem is not obeyed and the entropy decreases. We interpret this in terms of energy thermostat transactions, which damp out for long times. At long times, the system enters a very long relaxation at nearly fixed energy, the Boltzmann $H$ theorem is obeyed, and entropy increases. This is consistent with a system of nearly constant energy but varying order.

There are important differences between the analytic stat mech model studied in Sec. III and the relaxed 2D computer 
model in Sec. IV, which give complementary insights into the dislocation ordering problem. In the computer model, the full angular dependent force law is employed, so the frustration and noise is generated self-consistently by the system itself. Thus the dislocation temperature is not tunable- the heat bath is self-consistent with the physics of the dislocation interactions. However, in the analytic model, we separated the dislocation population into two subpopulations: One for the attractive orientations of the dislocations and one for the repulsive. The unperturbed energy functionals for these subpopulations do not possess interaction terms between the two populations, so the ground state of the system is fully ordered with half the dislocations in a single perfect wall and half in a regular lattice. But the fluctuations caused by the noise temperature are tunable, corresponding to turning on the interactions between the populations in a tunable manner. Thus the analytic model illustrates in a very graphic and intuitive manner the ordering physics in the dislocation system, even though the model is much more approximate and abstract than the computer model.

But our 2D computer modeling has addressed only an extremely simplified version of the dislocation problem. In particular, it is restricted to the 2D case, and there is only one Burgers vector present, so no annihilation takes place, and no dipoles are formed. Further, the relaxed structures found can be expected to be different from those in a steady state deformation, and it would be useful to explore the steady state deforming system in 2D.

If one asks how the ideas developed here for 2D dislocation systems might be extended to 3D, the first answer is that the general idea that noise and energetics drive the system in opposite directions is surely valid. But to extend the analytic model to 3D seems impossible for the same reason that no analytic dislocation theory can be extended into 3D. How to extend the ideas developed for the $2 \mathrm{D}$ simulations to $3 \mathrm{D}$ is more interesting. Although a direct implementation of a large $3 \mathrm{D}$ ensemble is impossible at present, the idea of a $3 \mathrm{D}$ en- semble of systems is certainly valid. Also, the noise function is easily extended to 3D. Likewise, it should be possible to define a 3D wall correlation function from which an energetic driving force could be defined. But such a 3D driving force will be weaker than the 2D analogue, because of competition from other 3D energetic driving forces. Nevertheless, the general concept seems possible, and the extension to other driving forces sounds to us like a fruitful direction to explore for understanding the very complex physics of metal deformation. Thus an extension to 3D seems possible in principle, but implementing it straightforwardly in practice seems beyond our current capabilities.

One alternative idea presents itself to us: Would an ensemble of simulations be necessary in 3D? Maybe not, because any single implementation of a 3D dislocation system may be sufficiently complex to incorporate enough of the statistical variation of an actual ensemble to be useful. But, if so, the 3D system would have to contain a large number of cells to generate the necessary variability-another challenge to current computer capabilities. But if such a proposition is valid, then 3D implementation of the ideas of this paper might be feasible. In any case, this is another question for later investigation.

The existence of a time dependent $H$ function as a means for studying dislocation structure evolution in combination with the twin ideas of ensemble defined driving forces and a noise induced effective temperature is the main vista we hope has been opened up in this paper. However, there are clearly many relevant directions yet to be explored.

\section{ACKNOWLEDGMENTS}

It is a pleasure to acknowledge the collaborative involvement of Richard LeSar in other aspects of this problem, and his comments on the current paper. It is also a pleasure to acknowledge discussions with Brad Holian, who was very helpful in explaining thermostat action.
*Electronic address: robbm@toast.net

${ }^{1}$ D. A. McQuarrie, Statistical Mechanics (Harper Collins, New York, 1979).

${ }^{2}$ D. J. Evans and G. P. Morriss, Statistical Mechanics of Nonequilibrium Liquids (Academic Press, New York, 1990).

${ }^{3}$ J. R. Dorfman, An Introduction to Chaos in Nonequilbrium Statistical Mechanics (Cambridge University Press, Cambridge, UK, 1999).

${ }^{4}$ L. Rondoni and E. G. D. Cohen, Nonlinearity 13, 1905 (2000).

${ }^{5}$ I. K. Ono, C. S. O'Hern, D. J. Durian, S. A. Langer, A. J. Liu, and S. R. Nagel, Phys. Rev. Lett. 89, 095703 (2002).

${ }^{6}$ C. S. O'Hern, A. J. Liu, and S. R. Nagel, Phys. Rev. Lett. 93, 165702 (2004).

${ }^{7}$ A. S. Argon, in Physical Metallurgy, edited by R. W. Cahn and P. Haasen (North-Holland, Amsterdam, 1996), Vol. 3, pp. 18771956.

${ }^{8}$ V. I. Berdichevski, J. Mech. Phys. Solids 53, 2457 (2005).
${ }^{9}$ V. I. Berdichevski, Thermodynamics of Chaos and Order (Chapman and Hall, New York, 1997).

${ }^{10}$ S. F. Edwards and D. V. Grinev, Phys. Rev. E 58, 4758 (1998).

${ }^{11}$ A. H. W. Ngan, Scr. Mater. 52, 1005 (2005).

${ }^{12}$ D. Kuhlmann-Wilsdorf, Mater. Res. Innovations 1, 265 (1998).

${ }^{13}$ P. Hähner, Appl. Phys. A: Mater. Sci. Process. 62, 473 (1996).

${ }^{14}$ P. Hähner, Scr. Mater. 47, 705 (2002).

${ }^{15}$ P. Hähner, K. Bay, and M. Zaiser, Phys. Rev. Lett. 81, 2470 (1998).

${ }^{16}$ R. Thomson, M. Koslowski, and R. LeSar, Phys. Rev. B 73, 024104 (2006).

${ }^{17}$ F. Reif, Fundamentals of Statistical and Thermal Physics (McGraw-Hill, New York, 1965).

${ }^{18}$ R. C. Tolman, The Principles of Statistical Mechanics (Oxford University Press, London, UK, 1938).

${ }^{19}$ B. L. Holian, A. F. Voter, and R. Ravelo, Phys. Rev. E 52, 2338 (1995). 